\title{
Treatment of Discoid Lupus Erythematosus in a Dog with Human Intravenous Immunoglobulin
}

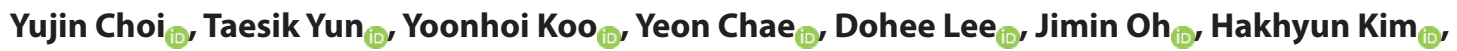 \\ Mhan-Pyo Yang \& Byeong-Teck Kang
}

\begin{abstract}
Background: Discoid lupus erythematosus (DLE) is a common canine autoimmune skin disease, in which systemic manifestations are absent. Skin Lesions are usually present on the nasal planum, and characterised by erythema, depigmentation, erosion, ulceration, and crusting. The diagnosis is based on histopathological results, which should demonstrate lymphoplasmacytic lichenoid-interface dermatitis. Human intravenous immunoglobulin (hIVIg) has been used in veterinary medicine to treat cutaneous diseases including erythema multiforme, PF, and severe adverse cutaneous drug reactions. In human medicine, it has been effective to treat DLE. This report firstly describes the clinical response to hIVIg in a dog with DLE resistant to common immunosuppressive drugs.

Case: A 5-year-old, intact female Shih Tzu presented with a 1-month history of slowly progressive black crusting on the nasal planum, chin, and claw. Based on the results of a dermatologic examination, superficial pyoderma was diagnosed. The skin lesions did not improve during and after anti-infective treatment. After removing the crusts, a skin biopsy was obtained from the muzzle. Histopathology of lesional skin biopsy specimens revealed lymphoplasmacytic interface dermatitis at the dermoepidermal junction. Microscopic examination also revealed vacuolar changes and pigmentary incontinence of the basal layer as a lichenoid tissue reaction. No mites or fungi were detected on the skin section. The absence of acantholytic cells excluded pemphigus foliaceus, which is also characterised by the lesions of the nasal planum. Based on the distribution of the lesions, histopathology and exclusion of other dermatoses, the dog was diagnosed with DLE. The skin lesions temporarily improved after treatment with prednisolone ( $2 \mathrm{mg} / \mathrm{kg}$ PO q12h). However, after tapering the dose of prednisolone, new black crusts developed on the nasal planum and claw. Although the dog was successively treated with other immunosuppressive drugs, including azathioprine, cyclosporin with dexamethasone, and mycophenolate mofetil, black crusts still remained. Due to the low efficacy of these immunosuppressive drugs, hIVIg was administered at $0.5 \mathrm{~g} / \mathrm{kg}$ once daily for 4 days, for a total dose of $2 \mathrm{~g} / \mathrm{kg}$. During hIVIg administration, the crusted lesions gradually improved. After the hIVIg administration, the dog was treated with prednisolone (1 mg/kg PO q12h). The lesions were almost in complete remission at 21 days after an additional application of prednisolone. The skin lesions did not recur, and the treatment was eventually discontinued after 6 weeks of additional prednisolone application.

Discussion: The standard treatment of canine DLE includes glucocorticoids, and second-line immunosuppressive drugs, such as azathioprine and cyclosporine, are usually added in cases resistant to steroids. This case suggests that hIVIg may be beneficial as an adjunctive treatment option for canine DLE, especially when the application of standard immunosuppressive drugs is limited due to adverse effects or low efficacy. There is evidence from several studies that the steroid-sparing effect of hIVIg is significant in human patients. In the current case, the effective dose of prednisolone was reduced to 2 $\mathrm{mg} / \mathrm{kg} / \mathrm{day}$ after hIVIg administration, and prednisolone therapy was finally discontinued completely. The hIVIg appears to lower the daily steroid dose requirement in this dog.
\end{abstract}

Keywords: autoimmune skin disease, discoid lupus erythematosus, canine dermatology, immunosuppressive drug, human intravenous immunoglobulin. 


\section{INTRODUCTION}

Discoid lupus erythematosus (DLE) is a common canine autoimmune skin disease, with skin lesions usually characterised by erythema, depigmentation, erosion, ulceration, and crusting of the nasal planum and surrounding skin [17]. Glucocorticoids are the first-line drugs for the treatment of DLE [14]. It is important to use alternative or adjunctive immunosuppressive therapy for clinical response enhancement and reduction of the adverse effects of steroids [22].

In human medicine, human intravenous immunoglobulin (hIVIg) is used in autoimmune and inflammatory diseases. Although data on the efficacy of hIVIg in human DLE are limited, it has been successfully used to treat human DLE [20]. Furthermore, hIVIg is considered as an adjunctive therapy in the management of dogs resistant to standard immunosuppressive protocols [22]. The hIVIg has been used in veterinary medicine to treat immune-mediated haemolytic anaemia, thrombocytopenia, and cutaneous diseases $[1,4,7,18]$. In 2006, Rahilly et al. [15] described the successful use of hIVIg to treat a dog with severe pemphigus foliaceus (PF), the most common canine immune-mediated skin disease. However, there are no reports on the use of hIVIg in canine DLE. This report firstly describes the clinical response to hIVIg in a dog with DLE resistant to common immunosuppressive drugs.

\section{CASE}

A 5-year-old, intact female Shih Tzu presented with a 1-month history of slowly progressive black crusting on the nasal planum, chin, and claw, as well as a 3-year history of pruritus on the face, ventral abdomen, four paws, and both ear pinnae (Figure 1). No other notable findings were observed on physical examination. Cytological examination of the lesions revealed bacterial infection, but no acantholytic cells. Scraping, trichogram, and Wood's lamp examination were unremarkable.

Based on the results of a dermatologic examination, superficial pyoderma was diagnosed. The initial differential diagnoses included deep pyoderma, autoimmune disease, atopic dermatitis, cutaneous adverse food reaction, demodicosis, and dermatophytosis. The dog was treated for 18 days with amoxicillin-clavulanate $^{1}$ [25 mg/kg PO q12h] and $2 \%$ chlorhexidine and miconazole shampoos ${ }^{2}$ [twice a week; Malaseb-F]. Escherichia coli was isolated from a swab of the skin lesions, and due to a lack of improvement, the dog was treated for 6 days with trimethoprim/sulfamethoxazole ${ }^{3}$ [30 mg/kg PO q12h], in accordance with the results of an antimicrobial susceptibility test. For the next 6 days, ivermectin ${ }^{4}$ [300 mcg/kg PO q24h; Ivomec] and itraconazole ${ }^{5}$ [10 mg/kg PO q24h; Sponazol Tab.] were added to differentiate demodicosis and dermatophytosis. However, the skin lesions did not improve during and after anti-infective treatment. Results of fungal cultures were also negative.

After removing the crusts, a skin biopsy was obtained from the muzzle. Histopathology of lesional skin biopsy specimens revealed mild epidermal hyperplasia and interface dermatitis at the dermoepidermal junction (Figure 2). The interface dermatitis was composed of lymphocytes and plasma cells. Microscopic examination also revealed vacuolar changes and pigmentary incontinence of the basal layer as a lichenoid tissue reaction. Crusts and inflammatory cells were seen on the epidermis. The crusts were mainly composed of neutrophils. No mites or fungi were detected on the skin section. The absence of acantholytic cells excluded PF, which is also characterised by the lesions of the nasal planum. Clinical features, histopathologic findings, and exclusion of other dermatoses supported the diagnosis of DLE.

Given the diagnosis of DLE, the dog was treated for 1 week with prednisolone ${ }^{6}[2 \mathrm{mg} / \mathrm{kg}$ PO q12h; Solondo Tab.] and topical hydrocortisone cre$\mathrm{am}^{7}$ [twice per day; Hydrocortisone Ointment]. This treatment resulted in a temporary improvement in the skin lesion. The prednisolone dose was reduced to $1.5 \mathrm{mg} / \mathrm{kg} \mathrm{q} 12 \mathrm{~h}$, and the skin lesions again worsened. Azathioprine $^{8}$ [2 mg/kg PO q24h, Immuthera Tab.] was added in the last week of prednisolone treatment due to the lack of a clinical response to the steroid; prednisolone was administered for a total of 1 month and stopped after tapering. Because new black crusts developed on the nasal planum and claw, cyclosporin ${ }^{9}$ [ $8 \mathrm{mg} / \mathrm{kg}$ PO q24h for 1 month, Cipol-N Soft Cap.] was initiated concurrently with dexamethasone ${ }^{6}[0.3 \mathrm{mg} / \mathrm{kg}$ PO q12h for 1 week, followed by $0.3 \mathrm{mg} / \mathrm{kg}$ PO q $24 \mathrm{~h}$ for 3 weeks; Dexamethasone Tab.]. The skin lesions were not improved after the successive prescriptions of azathioprine and cyclosporin with dexamethasone. Subsequently, the dog was treated for 1 month with mycophenolate mofetil ${ }^{10}[20 \mathrm{mg} / \mathrm{kg}$ PO q12h, Cellcept Cap.]; however, the skin lesions still did not improve. 


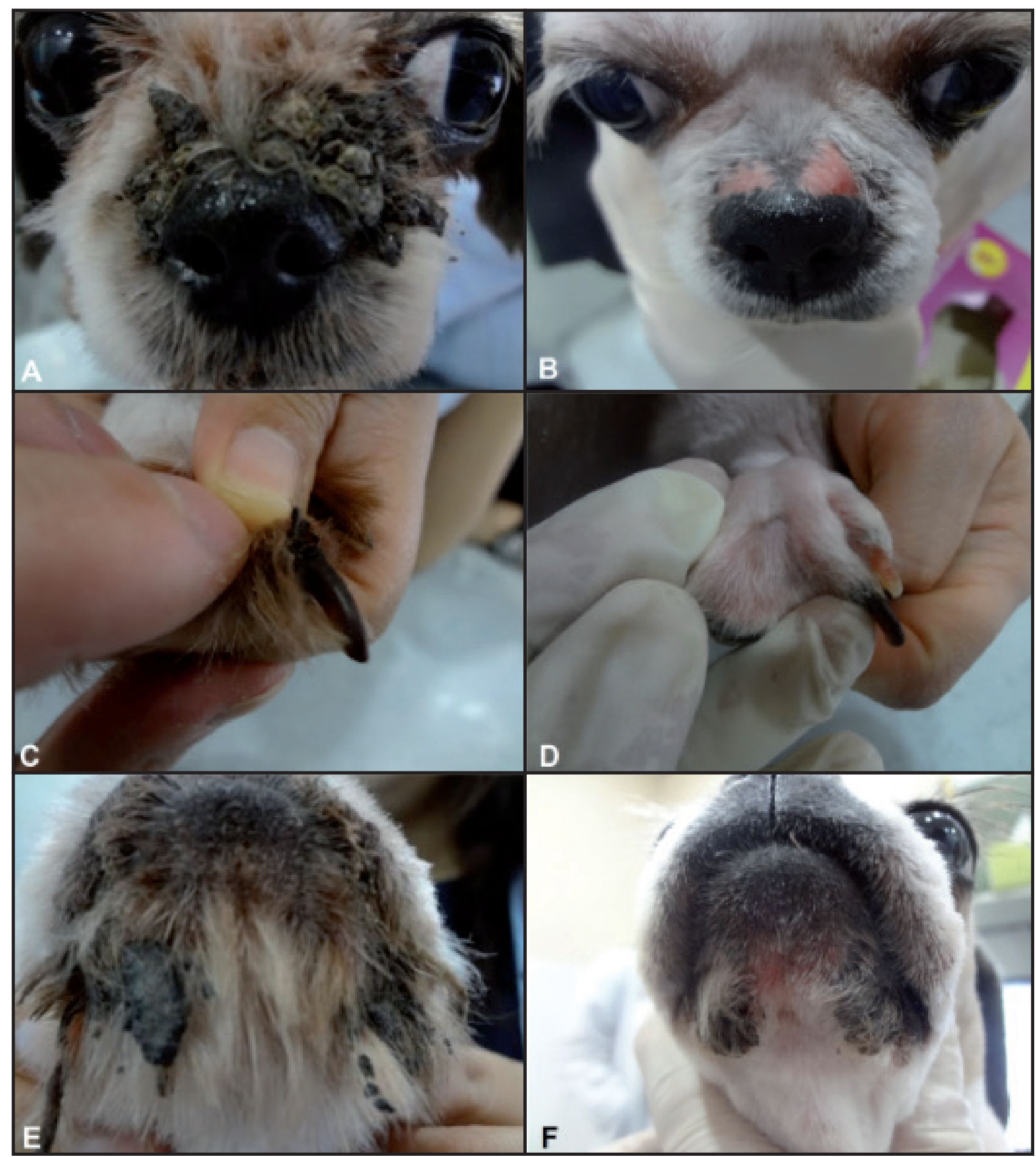

Figure 1. Comparison of the skin lesions, characterised by black crusts on the nasal planum, chin, and claw, between the first visit (A, C, E) and after hIVIg human intravenous immunoglobulin administration and additional prednisolone application (B, D, F).

Due to the insufficient clinical response after 3 months of immunosuppressive therapy, $\operatorname{hIVIg}^{11}$ (Liv gamma Inj.) was administered [0.5 g/ $/ \mathrm{kg}$ once daily for 4 days of hospitalisation, for a total dose of $2 \mathrm{~g} / \mathrm{kg}$ ]. After the hIVIg administration, the dog was treated with prednisolone (1 mg/kg PO q12h). Three weeks later, the black crust on the nasal planum was almost in complete remission (Figure 1). The lesions on the chin and claw were markedly decreased in severity, but some crust remained. The skin lesions did not recur, and the treatment was eventually discontinued after 6 weeks of additional prednisolone application. 


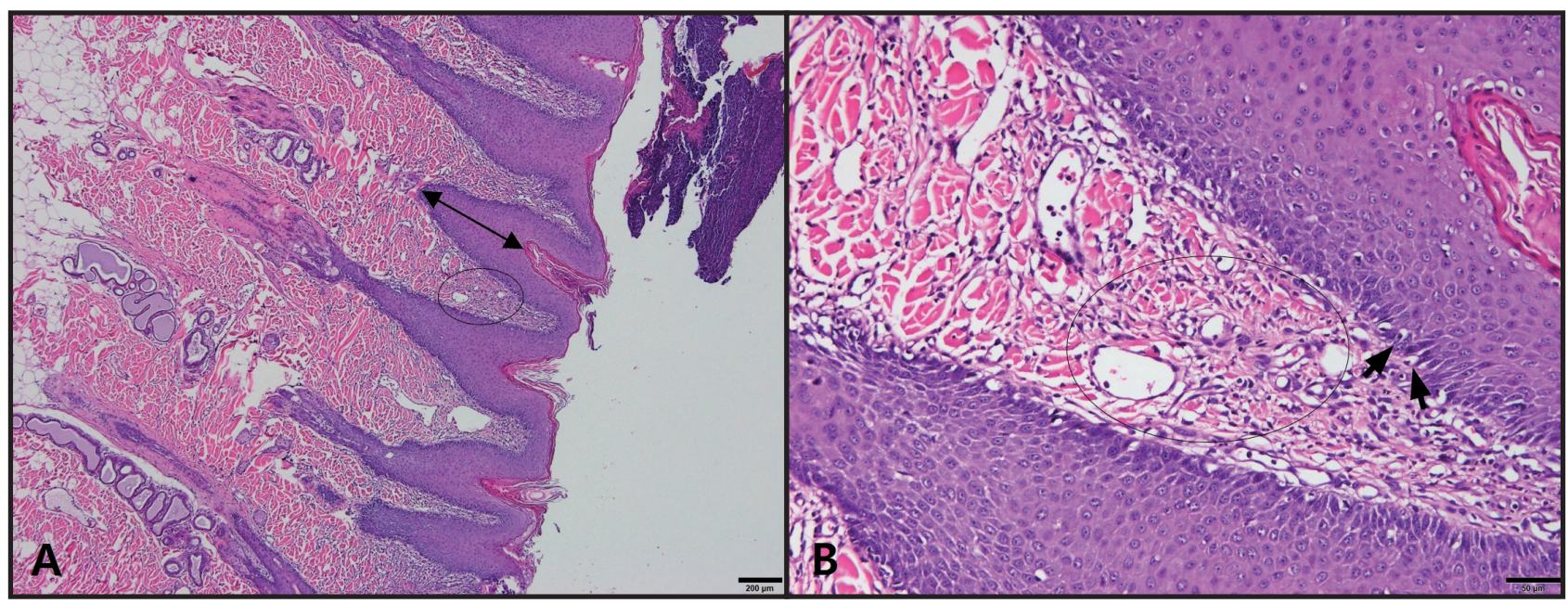

Figure 2. Histopathologic features of the skin biopsy obtained from the muzzle. Mild hyperplasia is observed in the epidermis (arrows). Inflammatory cells, composed of lymphocytes and plasma cells, infiltrated the dermoepidermal junction (circles). Note the vacuolar changes in the basal layer (arrow heads) [H\&E; A: 40x, B: 400x].

\section{DISCUSSION}

Discoid lupus erythematosus (DLE) is a relatively benign lupus erythematosus, in which systemic manifestations are absent [17]. Recently, DLE has been classified as the most common form of cutaneous lupus erythematosus. Lesions are usually present on the nasal planum, and also develop in the ear pinnae and periocular region [2]. The diagnosis of DLE is based on histopathological results, which should demonstrate lymphoplasmacytic lichenoid-interface dermatitis [2]. Additionally, direct immunofluorescence and immunohistochemistry reveal IgG and IgM deposits at the dermoepidermal junction in most cases [2]. In the current case, no attempt was made to confirm the diagnosis of DLE by direct immunofluorescence or immunohistochemistry. However, with reference to other DLE studies, the diagnosis was made based on the clinical appearance, histopathologic findings, and the exclusion of other dermatoses [2,12].

The standard treatment of canine DLE includes oral and/or topical glucocorticoids [14]. In cases resistant to steroids, second-line immunosuppressive drugs, such as azathioprine and cyclosporine, are usually added $[14,22]$. Combined tetracycline-niacinamide, topical $0.1 \%$ tacrolimus, and hydroxychloroquine can also be used as initial treatments [13,19,23]. In general, the prognosis of canine DLE is good, with appropriate treatment. However, relapses are common after medications are tapered [2].

In cases of human DLE refractory to first-line therapy, hIVIg has been employed [20]. The hIVIg is a blood product containing primarily $\mathrm{IgG}$, derived from the plasma of 1,000 to 60,000 human donors [8]. The hIVIg has been used in veterinary medicine to treat immune-mediated disorders, such as immune-mediated haemolytic anaemia, immune-mediated thrombocytopenia, myasthenia gravis, sudden acquired retinal degeneration, and cutaneous diseases $[1,4,7,18]$. Especially among dogs with cutaneous diseases, hIVIg is used in erythema multiforme, $\mathrm{PF}$, and severe adverse cutaneous drug reactions [5,15,21].

Nasal depigmentation, erythema, erosion, ulceration, crusts, and haemorrhage are characteristic in canine DLE [17]. The main skin lesions in the current case were black crusts on the nasal planum, which were very similar to those described in canine DLE. However, the other lesions showed some differences, including an unusual distribution (e.g. the chin and claw).

Although hIVIg is considered as an option for human patients with DLE refractory to standard therapies, many clinicians are unsure of the role of hIVIg in DLE [20]. However, various actions of hIVIg have been proposed; the most well described actions comprise complement inhibition, Fc receptor saturation, pathogenic autoantibody catabolism, and the regulation of cytokine release [8]. In the current case, no quantitative tests were performed to confirm the therapeutic mechanism of DLE.

The hIVIg appears to lower the daily steroid dose requirement. There is evidence from several studies that the steroid-sparing effect of hIVIg is significant in human patients $[3,10,24]$. In the current case, the effective dose of prednisolone was reduced to $2 \mathrm{mg} /$ 
$\mathrm{kg} /$ day after hIVIg administration, and prednisolone therapy was finally discontinued completely.

There is a risk of an acute hypersensitivity reaction to hIVIg administration in dogs due to the infusion of human-derived foreign proteins [1]. Other adverse effects reported in human medicine include thromboembolism, renal insufficiency, hypotension, and aseptic meningitis $[6,9,11,16]$. In the current case, no adverse effects were observed during and after hIVIg administration.

In conclusion, this report was the first to describe the clinical response to hIVIg in a dog with DLE. The hIVIg may be considered as an adjunctive treatment option for canine DLE, especially when the application of standard immunosuppressive drugs is limited due to adverse effects or low efficacy.

\author{
MANUFACTURERS \\ ${ }^{1}$ Aprogen Pharmaceuticals. Seongnam, South Korea. \\ ${ }^{2}$ Dermcare-Vet Pty Ltd. Slacks Creek, Australia. \\ ${ }^{3}$ Samil Pharmaceuticals. Seoul, South Korea. \\ ${ }^{4}$ Merial Saúde. Paulinia, SP, Brazil. \\ ${ }^{5}$ Nelson Korea Pharmaceutical Company. Eumseong, South Korea. \\ ${ }^{6}$ Yuhan Corporation. Seoul, South Korea. \\ ${ }^{7}$ Vivozon Pharmaceutical. Seoul, South Korea. \\ ${ }^{8}$ Celltrion Pharm Inc. Cheongju, South Korea. \\ ${ }^{9}$ Chong Kun Dang Pharmaceutical Corporation. Seoul, South Korea. \\ ${ }^{10}$ Roche Korea Co. Ltd. Seoul, South Korea. \\ ${ }^{11}$ SK Chemicals Co. Ltd. Seongnam, South Korea.
}

Acknowledgements. This work was supported by the National Research Foundation of Korea (NRF) grant funded by the Korea government (MSIT) [No. 2021R1A2C1012058].

Declaration of interest. The authors report no conflicts of interest. The authors alone are responsible for the content and writing of the paper.

\section{REFERENCES}

1 Abelson A.L., Shelton G.D., Whelan M.F., Cornejo L., Shaw S. \& O’Toole T.E. 2009. Use of mycophenolate mofetil as a rescue agent in the treatment of severe generalized myasthenia gravis in three dogs. Journal of Veterinary Emergency and Critical Care. 19(4): 369-374.

2 Banovic F., Linder K.E., Uri M., Rossi M.A. \& Olivry T. 2016. Clinical and microscopic features of generalized discoid lupus erythematosus in dogs (10 cases). Veterinary Dermatology. 27(6): 488-e131.

3 Baum S., Scope A., Barzilai A., Azizi E. \& Trau H. 2006. The role of IVIg treatment in severe pemphigus vulgaris. Journal of the European Academy of Dermatology and Venereology. 20(5): 548-52.

4 Bianco D., Armstrong P.J. \& Washabau R.J. 2007. Treatment of severe immune-mediated thrombocytopenia with human IV immunoglobulin in 5 dogs. Journal of Veterinary Internal Medicine. 21(4): 694-699.

5 Byrne K.P. \& Giger U. 2002. Use of human immunoglobulin for treatment of severe erythema multiforme in a cat. Journal of the American Veterinary Medical Association. 220(2): 197-201.

6 Cayco A.V., Perazella M.A. \& Hayslett J.P. 1997. Renal insufficiency after intravenous immune globulin therapy: a report of two cases and an analysis of the literature. Journal of the American Society of Nephrology. 8(11): 1788-1794.

7 Griffies J.D., Mendelsohn C.L., Rosenkrantz W.S., Muse R., Boord M.J. \& Griffin C.E. 2004. Topical 0.1\% tacrolimus for the treatment of discoid lupus erythematosus and pemphigus erythematosus in dogs. Journal of the American Animal Hospital Association. 40(1): 29-41.

8 Grozdanic S.D., Harper M.M. \& Kecova H. 2008. Antibody-mediated retinopathies in canine patients: mechanism, diagnosis, and treatment modalities. The Veterinary clinics of North America. Small animal practice. 38(2): 361-387.

9 Knezevic-Maramica I. \& Kruskall M.S. 2003. Intravenous immune globulins: an update for clinicians. Transfusion . 43(10): 1460-1480.

10 Kroez M., Kanzy E.-J., Gronski P. \& Dickneite G. 2003. Hypotension with intravenous immunoglobulin therapy: importance of $\mathrm{pH}$ and dimer formation. Biologicals. 31(4): 277-286.

11 Levy Y., Sherer Y., Ahmed A., Langevitz P., George J., Fabbrizzi F., Terryberry J., Meissner M., Lorber M., Peter J.B. \& Shoenfeld Y. 1999. A study of 20 SLE patients with intravenous immunoglobulin-clinical and serologic response. Lupus. 8(9): 705-712.

12 Marie I., Maurey G., Hervé F., Hellot M.F. \& Levesque H. 2006. Intravenous immunoglobulin-associated arterial and venous thrombosis; report of a series and review of the literature. British Journal of Dermatology. 155(4): 714-721.

13 Messinger L.2017. A Randomized, Double-Blinded Placebo Controlled Crossover Study Evaluating 0.03\% Tacrolimus Ointment Monotherapy in the Treatment of Discoid Lupus Erythematosus in Dogs. SOJ Veterinary Sciences. 3: 1-6. 
14 Oberkirchner U., Linder K.E. \& Olivry T. 2012. Successful treatment of a novel generalized variant of canine discoid lupus erythematosus with oral hydroxychloroquine. Veterinary Dermatology. 23(1): 65-70.

15 Olivry T., Alhaidari Z., Carlotti D.N., Guaguère E., Régnier A., Hubert B., Magnol J.P. \& Oksman F. 1987. Discoid lupus erythematosus in the dog: 22 cases [in French]. Pratique Médicale et Chirurgicale de l'Animal de Compagnie. 22: 205-214.

16 Rahilly L.J., Keating J.H. \& O'Toole T.E. 2006. The use of intravenous human immunoglobulin in treatment of severe pemphigus foliaceus in a dog. Journal of Veterinary Internal Medicine. 20(6): 1483-1486.

17 Scott D.W., Miller W.H. \& Griffin C.E. 2001. Autoimmune and Immune-Mediated Dermatoses In: Muller \& Kirk's Small Animal Dermatology. St. Louis: WB Saunders, pp.453-462.

18 Scott-Moncrieff J.C., Reagan W.J., Snyder P.W. \& Glickman L.T. 1997. Intravenous administration of human immune globulin in dogs with immune-mediated hemolytic anemia. Journal of the American Veterinary Medical Association. 210(11): 1623-1627.

19 Sekul E.A., Cupler E.J. \& Dalakas M.C. 1994. Aseptic meningitis associated with high-dose intravenous immunoglobulin therapy: frequency and risk factors. Annals of internal medicine. 121(4): 259-262.

20 Tenti S., Fabbroni M., Mancini V., Russo F., Galeazzi M. \& Fioravanti A. 2018. Intravenous Immunoglobulins as a new opportunity to treat discoid lupus erythematosus: A case report and review of the literature. Autoimmunity Reviews. 17(8): 791-795.

21 Trotman T.K., Phillips H., Fordyce H., King L.G., Morris D.O. \& Giger U. 2006. Treatment of severe adverse cutaneous drug reactions with human intravenous immunoglobulin in two dogs. Journal of the American Animal Hospital Association. 42(4): 312-320.

22 Viviano K.R. 2013. Update on immununosuppressive therapies for dogs and cats. The Veterinary Clinics of North America. Small Animal Practice. 43(5): 1149-1170.

23 White S.D., Rosychuk R.A., Reinke S.I. \& Paradis M. 1992. Use of tetracycline and niacinamide for treatment of autoimmune skin disease in 31 dogs. Journal of the American Veterinary Medical Association. 200(10): 1497-1500.

24 Zandman-Goddard G., Krauthammer A., Levy Y., Langevitz P. \& Shoenfeld Y. 2012. Long-term therapy with intravenous immunoglobulin is beneficial in patients with autoimmune diseases. Clinical Reviews in Allergy and Immunology. 42(2): 247-55. 